\title{
UK Renal Registry 15th Annual Report: Appendix F Additional Data Tables for 2011 New and Existing Patients
}

\section{F:1 Patients starting renal replacement therapy} in 2011

Table F.1.1. Number of patients on dialysis at 90 days (incident cohort $1 / 10 / 2010$ to $30 / 09 / 2011$ )

\begin{tabular}{lccccc}
\hline & \multicolumn{2}{c}{ Aged $<65$} & & \multicolumn{2}{c}{ Aged $\geqslant 65$} \\
\cline { 2 - 3 } \cline { 5 - 6 } & HD & PD & & HD & PD \\
& $\mathrm{N}$ & $\mathrm{N}$ & & $\mathrm{N}$ & $\mathrm{N}$ \\
\hline England & 1,676 & 675 & & 2,088 & 445 \\
N Ireland & 63 & 15 & & 87 & 14 \\
Scotland & 182 & 41 & & 182 & 31 \\
Wales & 104 & 50 & & 153 & 26 \\
UK & $\mathbf{2 , 0 2 5}$ & $\mathbf{7 8 1}$ & & $\mathbf{2 , 5 1 0}$ & $\mathbf{5 1 6}$ \\
\hline
\end{tabular}

Table F.1.3. First treatment modality (2011 incident cohort)

\begin{tabular}{llll}
\hline Centre & $\% \mathrm{HD}$ & $\% \mathrm{PD}$ & $\%$ transplant \\
\hline
\end{tabular}

\section{England}

B Heart

B QEH

Basldn

Bradfd

Brightn

Bristol

Camb

Carlis

Carsh

Chelms

Colchr

Covnt

Derby

Donc

Dorset

Dudley

Exeter

Glouc

Hull

Ipswi

85
73
83
79
76
74
68
41
78
72
100
65
52
79
66
61
80
76
54
59

Table F.1.2. Number of patients per treatment modality at 90 days (incident cohort 1/10/2010 to 30/09/2011)

\begin{tabular}{lrrrrr}
\hline & & & & Stopped \\
& HD & PD & Transplant & treatment & Died \\
\hline England & 3,764 & 1,120 & 482 & 20 & 317 \\
N Ireland & 150 & 29 & 9 & 2 & 7 \\
Scotland & 364 & 72 & 14 & 1 & 34 \\
Wales & 257 & 76 & 22 & 0 & 16 \\
UK & $\mathbf{4 , 5 3 5}$ & $\mathbf{1 , 2 9 7}$ & $\mathbf{5 2 7}$ & $\mathbf{2 3}$ & $\mathbf{3 7 4}$ \\
\hline
\end{tabular}

\begin{tabular}{lccc}
\hline Centre & \% HD & $\%$ PD & $\%$ transplant \\
\hline Prestn & 78 & 19 & 4 \\
Redng & 57 & 39 & 4 \\
Salford & 62 & 34 & 5 \\
Sheff & 79 & 14 & 7 \\
Shrew & 66 & 33 & 2 \\
Stevng & 79 & 15 & 5 \\
Sthend & 66 & 31 & 3 \\
Stoke & 72 & 27 & 1 \\
Sund & 76 & 18 & 5 \\
Truro & 74 & 21 & 5 \\
Wirral & 76 & 22 & 1 \\
Wolve & 68 & 31 & 1 \\
York & 75 & 18 & 8 \\
N Ireland & & & 14 \\
Antrim & 69 & 17 & 7 \\
Belfast & 81 & 12 & 3 \\
Newry & 76 & 21 & \\
Ulster & 94 & 6 & \\
West NI & 82 & 18 & \\
Scotland & & & \\
Abrdn & 88 & 12 &
\end{tabular}

UK Renal Registry, Southmead Hospital, Southmead Road, Bristol, BS10 5NB, UK

Email: renalregistry@renalregistry.nhs.uk

\section{KARGER}

Fax +4161306 1234

E-Mail karger@karger.ch

www.karger.com
(C) 2013 S. Karger AG, Basel

Accessible online at:

Accessible online at:
www.karger.com/nec 
Table F.1.3. Continued

\begin{tabular}{|c|c|c|c|c|c|c|c|}
\hline Centre & $\% \mathrm{HD}$ & $\% \mathrm{PD}$ & $\%$ transplant & Centre & $\% \mathrm{HD}$ & $\% \mathrm{PD}$ & $\%$ transplant \\
\hline Kent & 79 & 13 & 8 & Airdrie & 92 & 8 & \\
\hline L Barts & 68 & 25 & 7 & D \& Gall & 40 & 60 & \\
\hline L Guys & 77 & 9 & 15 & Dundee & 88 & 12 & \\
\hline L Kings & 73 & 25 & 2 & Dunfn & 79 & 21 & \\
\hline L Rfree & 73 & 18 & 10 & Edinb & 78 & 19 & 3 \\
\hline L St.G & 75 & 13 & 12 & Glasgw & 82 & 13 & 5 \\
\hline L West & 86 & 4 & 10 & Inverns & 83 & 17 & \\
\hline Leeds & 78 & 14 & 8 & Klmarnk & 64 & 36 & \\
\hline Leic & 69 & 20 & 12 & Wales & & & \\
\hline Liv Ain & 85 & 15 & & Bangor & 80 & 20 & \\
\hline Liv RI & 54 & 33 & 13 & Cardff & 75 & 19 & 6 \\
\hline M RI & 61 & 23 & 16 & Swanse & 78 & 22 & \\
\hline Middlbr & 84 & 9 & 7 & Wrexm & 73 & 19 & 8 \\
\hline Newc & 67 & 20 & 13 & England & 72 & 21 & 7 \\
\hline Norwch & 67 & 33 & & N Ireland & 81 & 14 & 5 \\
\hline Nottm & 63 & 33 & 4 & Scotland & 81 & 17 & 2 \\
\hline Oxford & 59 & 24 & 18 & Wales & 77 & 20 & 4 \\
\hline Plymth & 58 & 27 & 15 & UK & 73 & 20 & 7 \\
\hline Ports & 75 & 21 & 3 & & & & \\
\hline
\end{tabular}

Table F.1.4. First treatment modality, patient numbers (2011 incident cohort)

\begin{tabular}{lrrr}
\hline & HD & PD & Transplant \\
\hline England & 4,154 & 1,207 & 413 \\
N Ireland & 164 & 29 & 10 \\
Scotland & 402 & 82 & 11 \\
Wales & 267 & 69 & 13 \\
UK & $\mathbf{4 , 9 8 7}$ & $\mathbf{1 , 3 8 7}$ & $\mathbf{4 4 7}$ \\
\hline
\end{tabular}

Table F.1.5. Gender breakdown by treatment modality at 90 days (2011 incident cohort)

\begin{tabular}{|c|c|c|c|c|c|c|}
\hline \multirow[b]{2}{*}{ Centre } & \multicolumn{3}{|c|}{$\mathrm{HD}$} & \multicolumn{3}{|c|}{$\mathrm{PD}$} \\
\hline & $\%$ male & $\%$ female & $\mathrm{M}: \mathrm{F}$ ratio & $\%$ male & $\%$ female & $\mathrm{M}: \mathrm{F}$ ratio \\
\hline \multicolumn{7}{|l|}{ England } \\
\hline B Heart & 64 & 36 & 1.8 & 75 & 25 & 3.0 \\
\hline $\mathrm{B}$ QEH & 63 & 37 & 1.7 & 59 & 41 & 1.4 \\
\hline Basldn & 88 & 12 & 7.3 & 60 & 40 & 1.5 \\
\hline Bradfd & 61 & 39 & 1.6 & 63 & 38 & 1.7 \\
\hline Brightn & 73 & 27 & 2.6 & 63 & 38 & 1.7 \\
\hline Bristol & 61 & 39 & 1.6 & 65 & 35 & 1.8 \\
\hline Camb & 71 & 29 & 2.4 & 50 & 50 & 1.0 \\
\hline Carlis & 100 & & & 91 & 9 & 10.0 \\
\hline Carsh & 61 & 39 & 1.6 & 62 & 38 & 1.6 \\
\hline Chelms & 77 & 23 & 3.3 & 75 & 25 & 3.0 \\
\hline Colchr & 62 & 39 & 1.6 & & & \\
\hline Covnt & 73 & 27 & 2.7 & 79 & 21 & 3.8 \\
\hline Derby & 66 & 34 & 1.9 & 57 & 43 & 1.3 \\
\hline Donc & 64 & 36 & 1.7 & 75 & 25 & 3.0 \\
\hline Dorset & 60 & 40 & 1.5 & 61 & 39 & 1.6 \\
\hline Dudley & 64 & 36 & 1.8 & 54 & 46 & 1.2 \\
\hline Exeter & 66 & 34 & 1.9 & 67 & 33 & 2.0 \\
\hline Glouc & 71 & 30 & 2.4 & 64 & 36 & 1.7 \\
\hline Hull & 62 & 38 & 1.6 & 46 & 54 & 0.9 \\
\hline Ipswi & 71 & 29 & 2.4 & 70 & 30 & 2.3 \\
\hline Kent & 68 & 32 & 2.1 & 77 & 24 & 3.3 \\
\hline
\end{tabular}


Table F.1.5. Continued

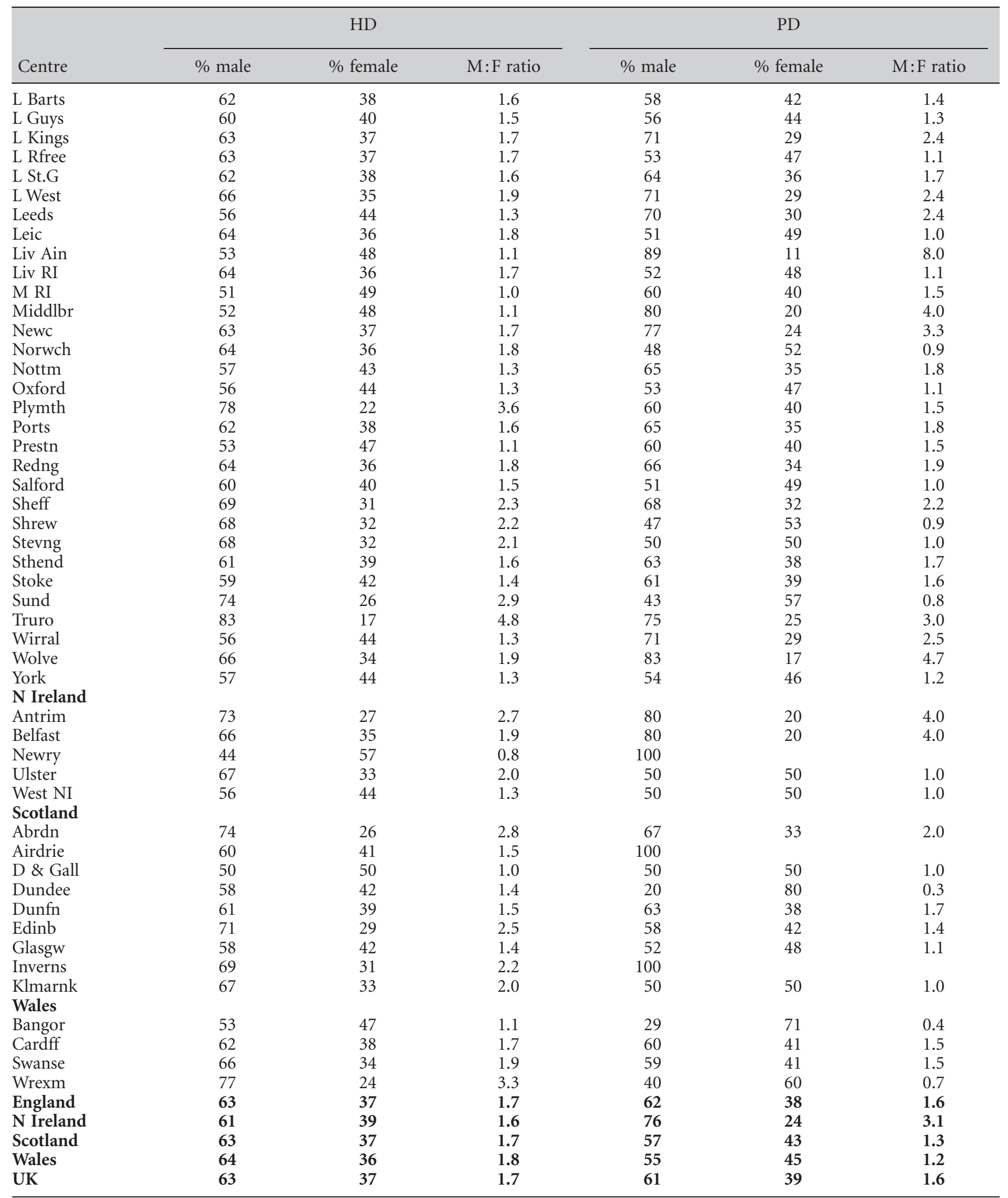


F:2 Prevalent patients on 31/12/2011

Table F.2.1. Treatment modalities for 2011 prevalent patients aged under and over 65

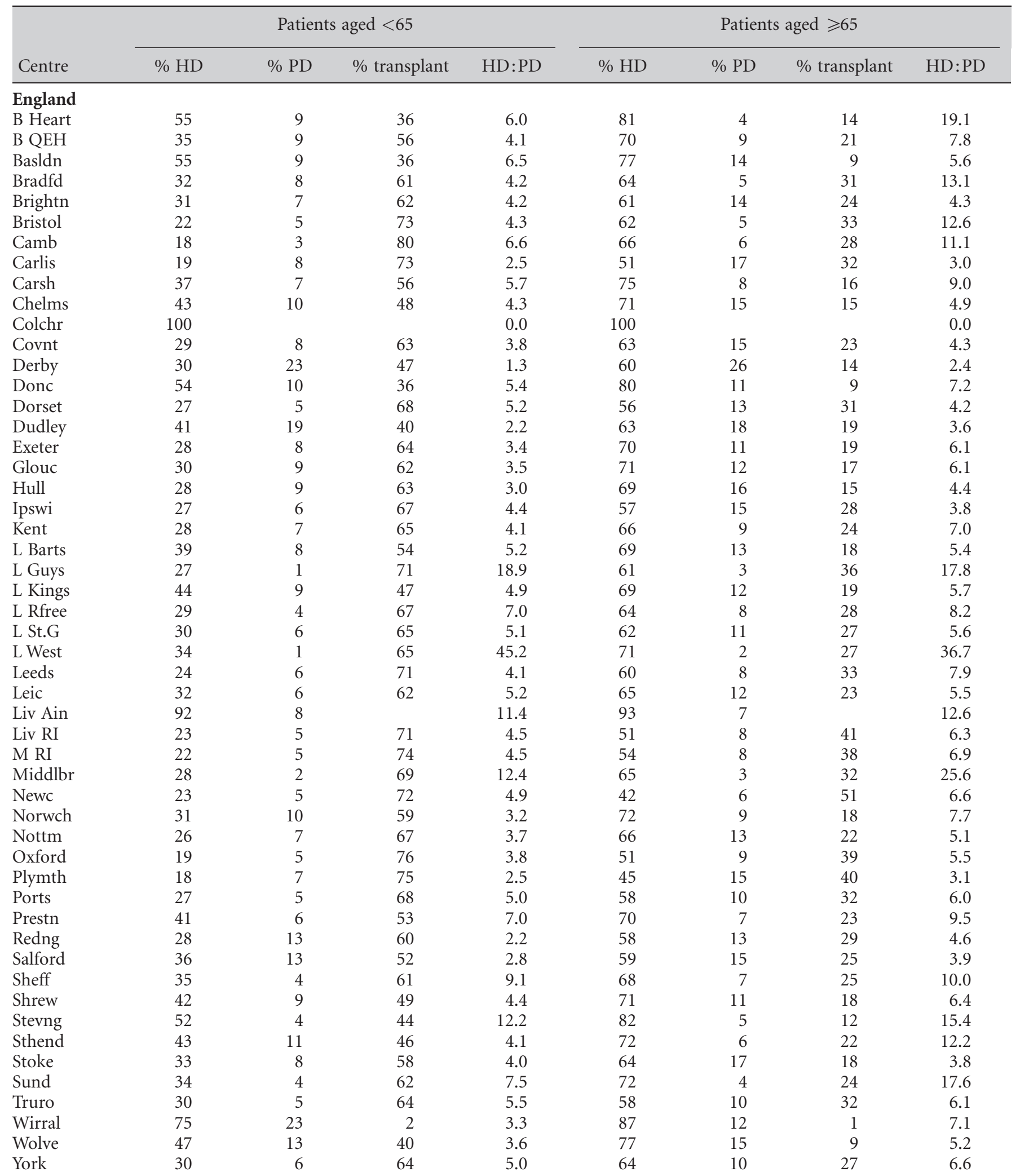


Table F.2.1. Continued

\begin{tabular}{|c|c|c|c|c|c|c|c|c|}
\hline \multirow[b]{2}{*}{ Centre } & \multicolumn{4}{|c|}{ Patients aged $<65$} & \multicolumn{4}{|c|}{ Patients aged $\geqslant 65$} \\
\hline & $\% \mathrm{HD}$ & $\% \mathrm{PD}$ & $\%$ transplant & $\mathrm{HD}: \mathrm{PD}$ & $\% \mathrm{HD}$ & $\% \mathrm{PD}$ & $\%$ transplant & HD:PD \\
\hline \multicolumn{9}{|l|}{$\mathrm{N}$ Ireland } \\
\hline Antrim & 41 & 5 & 54 & 7.5 & 77 & 7 & 16 & 10.9 \\
\hline Belfast & 24 & 4 & 72 & 6.4 & 58 & 6 & 36 & 9.6 \\
\hline Newry & 44 & 5 & 51 & 9.4 & 76 & 8 & 15 & 9.1 \\
\hline Ulster & 51 & 6 & 43 & 9.0 & 93 & & 7 & 0.0 \\
\hline West NI & 40 & 7 & 53 & 5.6 & 79 & 7 & 14 & 11.7 \\
\hline \multicolumn{9}{|l|}{ Scotland } \\
\hline Abrdn & 31 & 5 & 63 & 5.8 & 75 & 3 & 21 & 22.0 \\
\hline Airdrie & 41 & 3 & 56 & 14.3 & 74 & 3 & 23 & 24.3 \\
\hline D \& Gall & 31 & 5 & 64 & 6.0 & 57 & 23 & 20 & 2.5 \\
\hline Dundee & 32 & 5 & 63 & 6.3 & 66 & 6 & 28 & 10.7 \\
\hline Dunfn & 41 & 8 & 50 & 4.9 & 69 & 12 & 19 & 5.6 \\
\hline Edinb & 32 & 5 & 64 & 6.9 & 53 & 9 & 38 & 6.0 \\
\hline Glasgw & 32 & 3 & 66 & 10.8 & 66 & 4 & 30 & 15.7 \\
\hline Inverns & 22 & 6 & 72 & 3.8 & 72 & 13 & 15 & 5.4 \\
\hline Klmarnk & 35 & 14 & 52 & 2.5 & 74 & 17 & 8 & 4.3 \\
\hline \multicolumn{9}{|l|}{ Wales } \\
\hline Bangor & 82 & 18 & & 4.7 & 79 & 21 & & 3.8 \\
\hline Cardff & 20 & 5 & 75 & 4.1 & 58 & 10 & 32 & 5.6 \\
\hline Clwyd & 40 & 6 & 55 & 6.8 & 58 & 6 & 36 & 9.7 \\
\hline Swanse & 38 & 9 & 53 & 4.3 & 72 & 9 & 19 & 8.4 \\
\hline Wrexm & 26 & 8 & 66 & 3.1 & 59 & 9 & 33 & 6.9 \\
\hline England & 31 & 6 & 62 & 5.0 & 65 & 9 & 25 & 6.9 \\
\hline $\mathrm{N}$ Ireland & 33 & 5 & 62 & 6.8 & 73 & 6 & 21 & 12.6 \\
\hline Scotland & 33 & 5 & 63 & 6.7 & 67 & 8 & 26 & 8.7 \\
\hline Wales & 27 & 6 & 67 & 4.2 & 64 & 10 & 26 & 6.3 \\
\hline UK & 31 & 6 & 63 & 5.1 & 66 & 9 & 25 & 7.1 \\
\hline
\end{tabular}

Table F.2.2. Number of 2011 prevalent patients under and over 65 per treatment modality

\begin{tabular}{lrrrrrrrr}
\hline & \multicolumn{3}{c}{ Patients aged $<65$} & & \multicolumn{3}{c}{ Patients aged $\geqslant 65$} \\
\cline { 2 - 3 } \cline { 7 - 8 } & HD & PD & Transplant & & HD & PD & Transplant \\
\hline England & 9,030 & 1,795 & 18,029 & & 10,341 & 1,488 & 3,960 \\
N Ireland & 308 & 45 & 588 & & 417 & 33 & 119 \\
Scotland & 961 & 144 & 1,847 & & 917 & 105 & 350 \\
Wales & 453 & 108 & 1,114 & & 639 & 101 & 262 \\
UK & $\mathbf{1 0 , 7 5 2}$ & $\mathbf{2 , 0 9 2}$ & $\mathbf{2 1 , 5 7 8}$ & & $\mathbf{1 2 , 3 1 4}$ & $\mathbf{1 , 7 2 7}$ & $\mathbf{4 , 6 9 1}$ \\
\hline
\end{tabular}


Table F.2.3. Dialysis modalities for 2011 prevalent patients aged under 65

\begin{tabular}{|c|c|c|c|c|c|c|}
\hline Centre & $\begin{array}{c}\% \\
\text { home HD }\end{array}$ & $\begin{array}{c}\% \\
\text { hospital HD }\end{array}$ & $\begin{array}{c}\% \\
\text { satellite HD }\end{array}$ & $\begin{array}{c}\% \\
\text { CAPD }\end{array}$ & $\begin{array}{l}\% \text { unknown } \\
\text { type of PD }\end{array}$ & $\begin{array}{c}\% \\
\text { APD }\end{array}$ \\
\hline \multicolumn{7}{|l|}{ England } \\
\hline B Heart & 9 & 71 & 6 & 8 & 0 & 6 \\
\hline B QEH & 7 & 12 & 62 & 10 & 0 & 9 \\
\hline Basldn & 0 & 87 & 0 & 4 & 0 & 10 \\
\hline Bradfd & 0 & 73 & 8 & 2 & 0 & 18 \\
\hline Brightn & 11 & 38 & 32 & 7 & 0 & 12 \\
\hline Bristol & 11 & 16 & 54 & 7 & 0 & 12 \\
\hline Camb & 6 & 41 & 39 & 0 & 12 & 1 \\
\hline Carlis & 0 & 58 & 13 & 13 & 0 & 16 \\
\hline Carsh & 3 & 31 & 51 & 3 & 0 & 12 \\
\hline Chelms & 0 & 81 & 0 & 8 & 0 & 11 \\
\hline Colchr & 0 & 100 & 0 & 0 & 0 & 0 \\
\hline Covnt & 7 & 73 & 0 & 21 & 0 & 0 \\
\hline Derby & 5 & 52 & 0 & 29 & 0 & 14 \\
\hline Donc & 1 & 49 & 34 & 1 & 0 & 15 \\
\hline Dorset & 1 & 20 & 63 & 3 & 0 & 13 \\
\hline Dudley & 4 & 45 & 19 & 18 & 0 & 13 \\
\hline Exeter & 2 & 11 & 64 & 9 & 0 & 14 \\
\hline Glouc & 0 & 78 & 0 & 3 & 0 & 20 \\
\hline Hull & 4 & 35 & 35 & 10 & 0 & 15 \\
\hline Ipswi & 4 & 67 & 11 & 8 & 0 & 11 \\
\hline Kent & 8 & 29 & 44 & 20 & 0 & 0 \\
\hline L Barts & 2 & 35 & 47 & 5 & 0 & 11 \\
\hline L Guys & 10 & 23 & 62 & 2 & 0 & 3 \\
\hline L Kings & 1 & 21 & 60 & 4 & 0 & 13 \\
\hline L Rfree & 2 & 3 & 83 & 2 & 0 & 10 \\
\hline L St.G & 4 & 42 & 38 & 2 & 0 & 15 \\
\hline L West & 2 & 27 & 69 & 1 & 0 & 1 \\
\hline Leeds & 4 & 23 & 54 & 6 & 0 & 14 \\
\hline Leic & 6 & 19 & 59 & 6 & 0 & 11 \\
\hline Liv Ain & 0 & 24 & 68 & 0 & 0 & 8 \\
\hline Liv RI & 8 & 37 & 36 & 7 & 0 & 11 \\
\hline M RI & 18 & 31 & 32 & 4 & 0 & 14 \\
\hline Middlbr & 6 & 43 & 44 & 7 & 0 & 1 \\
\hline Newc & 11 & 72 & 0 & 3 & 0 & 14 \\
\hline Norwch & 8 & 45 & 23 & 19 & 0 & 4 \\
\hline Nottm & 11 & 38 & 30 & 10 & 0 & 11 \\
\hline Oxford & 5 & 36 & 37 & 5 & 0 & 16 \\
\hline Plymth & 7 & 64 & 0 & 21 & 1 & 7 \\
\hline Ports & 1 & 24 & 58 & 17 & 0 & 0 \\
\hline Prestn & 9 & 17 & 62 & 4 & 0 & 8 \\
\hline Redng & 0 & 30 & 39 & 31 & 0 & 1 \\
\hline Salford & 6 & 30 & 38 & 17 & 0 & 9 \\
\hline Sheff & 11 & 34 & 45 & 10 & 0 & 0 \\
\hline Shrew & 10 & 45 & 26 & 19 & 0 & 0 \\
\hline Stevng & 8 & 47 & 37 & 8 & 0 & 0 \\
\hline Sthend & 2 & 79 & 0 & 20 & 0 & 0 \\
\hline Stoke & 6 & 50 & 23 & 2 & 0 & 18 \\
\hline Sund & 2 & 68 & 19 & 7 & 0 & 5 \\
\hline Truro & 1 & 63 & 21 & 3 & 0 & 13 \\
\hline Wirral & 0 & 61 & 16 & 3 & 0 & 20 \\
\hline Wolve & 5 & 24 & 49 & 22 & 0 & 0 \\
\hline York & 9 & 53 & 22 & 17 & 0 & 0 \\
\hline \multicolumn{7}{|c|}{$\mathrm{N}$ Ireland } \\
\hline Antrim & 8 & 80 & 0 & 2 & 0 & 10 \\
\hline Belfast & 8 & 79 & 0 & 1 & 1 & 12 \\
\hline Newry & 2 & 88 & 0 & 0 & 0 & 10 \\
\hline
\end{tabular}


Table F.2.3. Continued

\begin{tabular}{|c|c|c|c|c|c|c|}
\hline Centre & $\begin{array}{c}\% \\
\text { home HD }\end{array}$ & $\begin{array}{c}\% \\
\text { hospital HD }\end{array}$ & $\begin{array}{c}\% \\
\text { satellite HD }\end{array}$ & $\begin{array}{c}\% \\
\text { CAPD }\end{array}$ & $\begin{array}{l}\% \text { unknown } \\
\text { type of PD }\end{array}$ & $\begin{array}{c}\% \\
\text { APD }\end{array}$ \\
\hline Ulster & 13 & 77 & 0 & 0 & 0 & 10 \\
\hline West NI & 4 & 81 & 0 & 1 & 0 & 14 \\
\hline \multicolumn{7}{|l|}{ Scotland ${ }^{*}$} \\
\hline Abrdn & 3 & 82 & 0 & 5 & 0 & 10 \\
\hline Airdrie & 0 & 93 & 0 & 3 & 0 & 4 \\
\hline D \& Gall & 4 & 82 & 0 & 4 & 0 & 11 \\
\hline Dundee & 0 & 86 & 0 & 3 & 0 & 10 \\
\hline Dunfn & 0 & 83 & 0 & 0 & 0 & 17 \\
\hline Edinb & 3 & 84 & 0 & 3 & 0 & 10 \\
\hline Glasgw & 8 & 83 & 0 & 2 & 0 & 6 \\
\hline Inverns & 9 & 70 & 0 & 9 & 0 & 12 \\
\hline Klmarnk & 4 & 67 & 0 & 2 & 0 & 26 \\
\hline \multicolumn{7}{|l|}{ Wales } \\
\hline Bangor & 22 & 47 & 14 & 4 & 0 & 14 \\
\hline Cardff & 11 & 22 & 47 & 14 & 0 & 6 \\
\hline Clwyd & 8 & 79 & 0 & 10 & 0 & 3 \\
\hline Swanse & 11 & 47 & 23 & 14 & 0 & 5 \\
\hline Wrexm & 2 & 68 & 6 & 25 & 0 & 0 \\
\hline England & 6 & 35 & 43 & 8 & 0 & 9 \\
\hline N Ireland & 7 & 81 & 0 & 1 & 0 & 12 \\
\hline Scotland & 4 & 83 & 0 & 3 & 0 & 10 \\
\hline Wales & 11 & 40 & 30 & 14 & 0 & 6 \\
\hline UK & 6 & 40 & 38 & 8 & 0 & 9 \\
\hline
\end{tabular}

${ }^{*}$ All haemodialysis patients in centres in Scotland are shown as receiving treatment at home or in centre as no data is available regarding satellite dialysis

Table F.2.4. Dialysis modalities for 2011 prevalent patients aged over 65

\begin{tabular}{|c|c|c|c|c|c|c|}
\hline Centre & $\begin{array}{c}\% \\
\text { home HD }\end{array}$ & $\begin{array}{c}\% \\
\text { hospital HD }\end{array}$ & $\begin{array}{c}\% \\
\text { satellite HD }\end{array}$ & $\begin{array}{c}\% \\
\text { CAPD }\end{array}$ & $\begin{array}{l}\% \text { unknown } \\
\text { type of PD }\end{array}$ & $\begin{array}{c}\% \\
\mathrm{APD}\end{array}$ \\
\hline
\end{tabular}

\section{England}

B Heart

B QEH

Basldn

Bradfd

Brightn

Bristol

Camb

Carlis

Carsh

Chelms

Colchr

Covnt

Derby

Donc

Dorset

Dudley

Exeter

Glouc

Hull

Ipswi

Kent

L Barts

L Guys

L Kings

L Rfree 
Table F.2.4. Continued

\begin{tabular}{|c|c|c|c|c|c|c|}
\hline Centre & $\begin{array}{c}\% \\
\text { home HD }\end{array}$ & $\begin{array}{c}\% \\
\text { hospital HD }\end{array}$ & $\begin{array}{c}\% \\
\text { satellite HD }\end{array}$ & $\begin{array}{c}\% \\
\text { CAPD }\end{array}$ & $\begin{array}{l}\% \text { unknown } \\
\text { type of PD }\end{array}$ & $\begin{array}{c}\% \\
\text { APD }\end{array}$ \\
\hline L St.G & 0 & 41 & 44 & 5 & 1 & 9 \\
\hline L West & 0 & 22 & 75 & 1 & 0 & 1 \\
\hline Leeds & 0 & 16 & 73 & 3 & 0 & 8 \\
\hline Leic & 2 & 16 & 67 & 6 & 0 & 9 \\
\hline Liv Ain & 0 & 16 & 77 & 2 & 0 & 5 \\
\hline Liv RI & 2 & 37 & 47 & 8 & 0 & 5 \\
\hline M RI & 2 & 31 & 54 & 2 & 0 & 10 \\
\hline Middlbr & 2 & 24 & 71 & 4 & 0 & 0 \\
\hline Newc & 1 & 86 & 0 & 3 & 0 & 10 \\
\hline Norwch & 2 & 49 & 38 & 9 & 0 & 3 \\
\hline Nottm & 3 & 40 & 41 & 9 & 0 & 7 \\
\hline Oxford & 2 & 39 & 44 & 6 & 0 & 9 \\
\hline Plymth & 0 & 75 & 0 & 22 & 0 & 3 \\
\hline Ports & 0 & 19 & 67 & 14 & 0 & 0 \\
\hline Prestn & 3 & 17 & 71 & 3 & 0 & 7 \\
\hline Redng & 1 & 43 & 38 & 17 & 0 & 1 \\
\hline Salford & 2 & 33 & 45 & 13 & 0 & 7 \\
\hline Sheff & 2 & 39 & 50 & 9 & 0 & 0 \\
\hline Shrew & 0 & 53 & 34 & 14 & 0 & 0 \\
\hline Stevng & 2 & 49 & 43 & 6 & 0 & 0 \\
\hline Sthend & 3 & 90 & 0 & 8 & 0 & 0 \\
\hline Stoke & 2 & 58 & 20 & 6 & 0 & 15 \\
\hline Sund & 0 & 67 & 28 & 2 & 0 & 3 \\
\hline Truro & 0 & 41 & 45 & 6 & 0 & 8 \\
\hline Wirral & 0 & 69 & 19 & 2 & 0 & 10 \\
\hline Wolve & 0 & 22 & 61 & 16 & 0 & 0 \\
\hline York & 0 & 54 & 33 & 13 & 0 & 0 \\
\hline \multicolumn{7}{|l|}{ N Ireland } \\
\hline Antrim & 0 & 92 & 0 & 3 & 0 & 5 \\
\hline Belfast & 3 & 87 & 0 & 2 & 0 & 8 \\
\hline Newry & 3 & 87 & 0 & 0 & 0 & 10 \\
\hline Ulster & 0 & 100 & 0 & 0 & 0 & 0 \\
\hline West NI & 1 & 91 & 0 & 0 & 0 & 8 \\
\hline \multicolumn{7}{|l|}{ Scotland ${ }^{*}$} \\
\hline Abrdn & 0 & 96 & 0 & 4 & 0 & 0 \\
\hline Airdrie & 0 & 96 & 0 & 0 & 0 & 4 \\
\hline D \& Gall & 0 & 71 & 0 & 9 & 0 & 20 \\
\hline Dundee & 0 & 91 & 0 & 1 & 0 & 8 \\
\hline Dunfn & 0 & 85 & 0 & 0 & 0 & 15 \\
\hline Edinb & 0 & 86 & 0 & 5 & 0 & 9 \\
\hline Glasgw & 1 & 93 & 0 & 2 & 0 & 4 \\
\hline Inverns & 2 & 83 & 0 & 5 & 0 & 10 \\
\hline Klmarnk & 3 & 78 & 0 & 2 & 0 & 17 \\
\hline \multicolumn{7}{|l|}{ Wales } \\
\hline Bangor & 3 & 57 & 19 & 7 & 0 & 14 \\
\hline Cardff & 1 & 13 & 71 & 11 & 0 & 4 \\
\hline Clwyd & 3 & 88 & 0 & 9 & 0 & 0 \\
\hline Swanse & 4 & 52 & 34 & 8 & 0 & 2 \\
\hline Wrexm & 0 & 84 & 4 & 13 & 0 & 0 \\
\hline England & 1 & 36 & 51 & 7 & 0 & 5 \\
\hline $\mathrm{N}$ Ireland & 2 & 91 & 0 & 1 & 0 & 6 \\
\hline Scotland & 1 & 89 & 0 & 3 & 0 & 8 \\
\hline Wales & 2 & 38 & 46 & 10 & 0 & 4 \\
\hline UK & 1 & 41 & 45 & 7 & 0 & 5 \\
\hline
\end{tabular}

${ }^{\star}$ All haemodialysis patients in centres in Scotland are shown as receiving treatment at home or in centre as no data is available regarding satellite dialysis 
Table F.2.5. Prevalent patients 2011, age ranges by centre (\%)

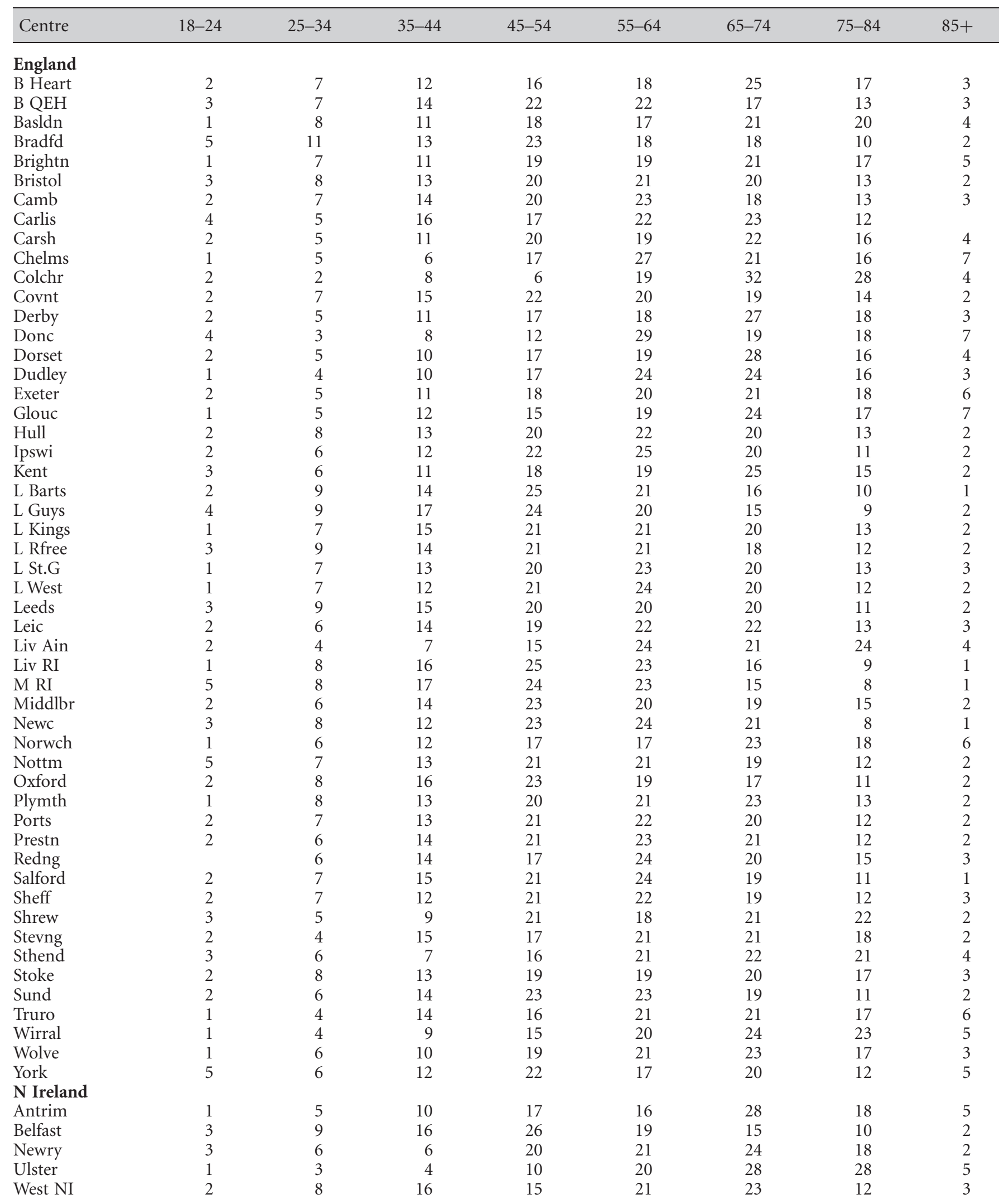


Table F.2.5. Continued

\begin{tabular}{lrrrrrrrr}
\hline Centre & $18-24$ & $25-34$ & $35-44$ & $45-54$ & $55-64$ & $65-74$ & $75-84$ & $85+$ \\
\hline Scotland & & & & & & & & \\
Abrdn & 4 & 10 & 15 & 18 & 24 & 18 & 11 & 1 \\
Airdrie & 2 & 10 & 16 & 20 & 23 & 15 & 13 & 1 \\
D \& Gall & 4 & 2 & 11 & 25 & 21 & 19 & 13 & 4 \\
Dundee & 1 & 5 & 17 & 18 & 20 & 21 & 15 & 5 \\
Dunfn & 2 & 6 & 12 & 19 & 21 & 23 & 16 & 2 \\
Edinb & 2 & 9 & 16 & 26 & 22 & 17 & 8 & 1 \\
Glasgw & 3 & 6 & 15 & 23 & 23 & 18 & 11 & 1 \\
Inverns & 2 & 5 & 16 & 27 & 20 & 13 & 14 & 3 \\
Klmarnk & 1 & 5 & 13 & 24 & 21 & 19 & 14 & 3 \\
Wales & 2 & 5 & 6 & 14 & 21 & 28 & 22 & 4 \\
Bangor & 3 & 7 & 14 & 21 & 23 & 18 & 11 & 3 \\
Cardff & 1 & 8 & 9 & 18 & 26 & 22 & 13 & 1 \\
Clwyd & 2 & 5 & 10 & 14 & 22 & 24 & 18 & 5 \\
Swanse & 5 & 7 & 14 & 20 & 19 & 17 & 16 & 1 \\
Wrexm & $\mathbf{2}$ & $\mathbf{7}$ & $\mathbf{1 3}$ & $\mathbf{2 1}$ & $\mathbf{2 1}$ & $\mathbf{2 0}$ & $\mathbf{1 3}$ & $\mathbf{3}$ \\
England & $\mathbf{2}$ & $\mathbf{7}$ & $\mathbf{1 3}$ & $\mathbf{2 1}$ & $\mathbf{1 9}$ & $\mathbf{2 1}$ & $\mathbf{1 4}$ & $\mathbf{3}$ \\
N Ireland & $\mathbf{2}$ & $\mathbf{7}$ & $\mathbf{1 5}$ & $\mathbf{2 2}$ & $\mathbf{2 2}$ & $\mathbf{1 8}$ & $\mathbf{1 2}$ & $\mathbf{2}$ \\
Scotland & $\mathbf{2}$ & $\mathbf{7}$ & $\mathbf{1 2}$ & $\mathbf{1 9}$ & $\mathbf{2 2}$ & $\mathbf{2 0}$ & $\mathbf{1 4}$ & $\mathbf{3}$ \\
Wales & $\mathbf{2}$ & $\mathbf{7}$ & $\mathbf{1 3}$ & $\mathbf{2 1}$ & $\mathbf{2 1}$ & $\mathbf{2 0}$ & $\mathbf{1 3}$ & $\mathbf{3}$ \\
UK & & & & & & & \\
\hline
\end{tabular}

Table F.2.6. Dialysis modalities for 2011 prevalent non-diabetic patients (all ages)

\begin{tabular}{|c|c|c|c|c|c|c|}
\hline Centre & $\begin{array}{c}\% \\
\text { home HD }\end{array}$ & $\begin{array}{c}\% \\
\text { hospital HD }\end{array}$ & $\begin{array}{c}\% \\
\text { satellite HD }\end{array}$ & $\begin{array}{c}\% \\
\text { CAPD }\end{array}$ & $\begin{array}{l}\% \text { unknown } \\
\text { type of PD }\end{array}$ & $\begin{array}{c}\% \\
\mathrm{APD}\end{array}$ \\
\hline
\end{tabular}

\section{England}

B Heart

B QEH

Basldn

Bradfd

Brightn

Bristol

Camb

Carlis

Carsh

Chelms

Covnt

Derby

Donc

Dorset

Dudley

Exeter

Glouc

Hull

Ipswi

Kent

L Barts

L Guys

L Kings

L St.G

L West

Leeds

Leic

Liv Ain

$\begin{array}{ll}4 & 78 \\ 5 & 10 \\ 0 & 87 \\ 0 & 65 \\ 6 & 43 \\ 7 & 13 \\ 3 & 38 \\ 0 & 53 \\ 2 & 32 \\ 0 & 79 \\ 3 & 77 \\ 6 & 60 \\ 0 & 47 \\ 1 & 20 \\ 2 & 47 \\ 1 & 10 \\ 1 & 84 \\ 3 & 37 \\ 3 & 66 \\ 5 & 28 \\ 1 & 31 \\ 7 & 21 \\ 2 & 19 \\ 2 & 40 \\ 1 & 24 \\ 2 & 19 \\ 4 & 17 \\ 3 & 13\end{array}$

$\begin{array}{rr}78 & 8 \\ 10 & 67\end{array}$

87

1

17

33

66

49

24

54

0

$0 \quad 20$

$0 \quad 26$

41

61

26

71

0

41

11

52

50

66

64

41

72

63

63

13

10
6
2
8
7
0
15
3
12
20
26
0
6
15
10
4
8
12
15
6
3
6
5
1
5
6
1

0
0
0
0
0
0
10
0
0
1
0
0
0
0
0
0
0
0
0
0
0
0
0
0
0
0
0
0

3

8

6

9

6

6
0

8

9

8

0
8
12

8
12

12

10

9

12

12

9
0

11

3

9

12

$\begin{array}{ll}9 & 63 \\ 17 & 63\end{array}$


Table F.2.6. Continued

\begin{tabular}{|c|c|c|c|c|c|c|}
\hline Centre & $\begin{array}{c}\% \\
\text { home HD }\end{array}$ & $\begin{array}{c}\% \\
\text { hospital HD }\end{array}$ & $\begin{array}{c}\% \\
\text { satellite HD }\end{array}$ & $\begin{array}{c}\% \\
\text { CAPD }\end{array}$ & $\begin{array}{l}\% \text { unknown } \\
\text { type of PD }\end{array}$ & $\begin{array}{c}\% \\
\mathrm{APD}\end{array}$ \\
\hline Liv RI & 6 & 37 & 41 & 8 & 0 & 8 \\
\hline M RI & 14 & 26 & 43 & 4 & 0 & 14 \\
\hline Middlbr & 4 & 31 & 59 & 5 & 0 & 0 \\
\hline Newc & 7 & 76 & 0 & 3 & 0 & 13 \\
\hline Norwch & 5 & 46 & 32 & 13 & 0 & 4 \\
\hline Nottm & 7 & 35 & 39 & 10 & 0 & 9 \\
\hline Oxford & 4 & 38 & 41 & 6 & 0 & 12 \\
\hline Plymth & 4 & 70 & 0 & 20 & 1 & 5 \\
\hline Ports & 1 & 19 & 65 & 16 & 0 & 0 \\
\hline Prestn & 7 & 15 & 66 & 4 & 0 & 8 \\
\hline Redng & 0 & 38 & 37 & 24 & 0 & 0 \\
\hline Salford & 6 & 36 & 38 & 11 & 0 & 9 \\
\hline Sheff & 7 & 37 & 47 & 9 & 0 & 0 \\
\hline Shrew & 5 & 49 & 30 & 15 & 0 & 0 \\
\hline Stevng & 6 & 48 & 38 & 8 & 0 & 0 \\
\hline Sthend & 2 & 85 & 0 & 14 & 0 & 0 \\
\hline Stoke & 4 & 53 & 24 & 5 & 0 & 15 \\
\hline Sund & 1 & 66 & 24 & 5 & 0 & 4 \\
\hline Truro & 0 & 46 & 37 & 4 & 0 & 13 \\
\hline Wirral & 0 & 71 & 18 & 0 & 0 & 11 \\
\hline Wolve & 3 & 20 & 57 & 20 & 0 & 0 \\
\hline York & 5 & 53 & 26 & 16 & 0 & 0 \\
\hline \multicolumn{7}{|l|}{ N Ireland } \\
\hline Antrim & 2 & 85 & 0 & 3 & 0 & 9 \\
\hline Belfast & 6 & 83 & 0 & 1 & 0 & 10 \\
\hline Newry & 2 & 86 & 0 & 0 & 0 & 12 \\
\hline Ulster & 5 & 93 & 0 & 0 & 0 & 2 \\
\hline West NI & 3 & 86 & 0 & 1 & 0 & 11 \\
\hline \multicolumn{7}{|l|}{ Scotland ${ }^{*}$} \\
\hline Abrdn & 2 & 88 & 0 & 5 & 0 & 5 \\
\hline Airdrie & 0 & 94 & 0 & 2 & 0 & 4 \\
\hline D \& Gall & 2 & 77 & 0 & 8 & 0 & 13 \\
\hline Dundee & 0 & 87 & 0 & 2 & 0 & 11 \\
\hline Dunfn & 0 & 83 & 0 & 0 & 0 & 17 \\
\hline Edinb & 2 & 84 & 0 & 4 & 0 & 10 \\
\hline Glasgw & 6 & 87 & 0 & 2 & 0 & 5 \\
\hline Inverns & 5 & 73 & 0 & 9 & 0 & 13 \\
\hline Klmarnk & 4 & 72 & 0 & 2 & 0 & 21 \\
\hline \multicolumn{7}{|l|}{ Wales } \\
\hline Bangor & 13 & 52 & 14 & 7 & 0 & 14 \\
\hline Cardff & 6 & 17 & 59 & 12 & 0 & 6 \\
\hline Clwyd & 4 & 85 & 0 & 9 & 0 & 2 \\
\hline Swanse & 7 & 49 & 30 & 11 & 0 & 3 \\
\hline Wrexm & 0 & 76 & 4 & 19 & 0 & 0 \\
\hline England & 4 & 36 & 46 & 8 & 0 & 7 \\
\hline $\mathrm{N}$ Ireland & 4 & 86 & 0 & 1 & 0 & 9 \\
\hline Scotland & 3 & 84 & 0 & 3 & 0 & 9 \\
\hline Wales & 6 & 39 & 38 & 12 & 0 & 5 \\
\hline UK & 4 & 41 & 40 & 7 & 0 & 7 \\
\hline
\end{tabular}

Excluded centres with $\geqslant 40 \%$ primary renal diagnosis aetiology uncertain (Colchester) as well as centres with $\geqslant 50 \%$ primary renal diagnosis not sent (London Royal Free)

Diabetic patients are patients with a primary renal disease code of diabetes

Non-diabetic patients are calculated as all patients excluding patients with diabetes and patients with a missing primary renal disease code

${ }^{*}$ All haemodialysis patients in centres in Scotland are shown as receiving treatment at home or in centre as no data is available regarding satellite dialysis 
Table F.2.7. Number of 2011 prevalent patients without diabetes by treatment modality

\begin{tabular}{lrrr}
\hline & HD & PD & Transplant \\
\hline England & 14,001 & 2,448 & 18,535 \\
N Ireland & 558 & 65 & 648 \\
Scotland & 1,484 & 212 & 2,005 \\
Wales & 850 & 166 & 1,209 \\
UK & $\mathbf{1 6 , 8 9 3}$ & $\mathbf{2 , 8 9 1}$ & $\mathbf{2 2 , 3 9 7}$ \\
\hline
\end{tabular}

Excluded centres with $\geqslant 40 \%$ primary renal diagnosis aetiology uncertain (Colchester) as well as centres with $\geqslant 50 \%$ primary renal diagnosis not sent (London Royal Free)

Diabetic patients are patients with a primary renal disease code of diabetes

Non-diabetic patients are calculated as all patients excluding patients with diabetes and patients with a missing primary renal disease code

Table F.2.8. Dialysis modalities for 2011 prevalent patients without diabetes aged under 65

\begin{tabular}{|c|c|c|c|c|c|c|}
\hline Centre & $\begin{array}{c}\% \\
\text { home HD }\end{array}$ & $\begin{array}{c}\% \\
\text { hospital HD }\end{array}$ & $\begin{array}{c}\% \\
\text { satellite HD }\end{array}$ & $\begin{array}{c}\% \\
\text { CAPD }\end{array}$ & $\begin{array}{l}\% \text { unknown } \\
\text { type of PD }\end{array}$ & $\begin{array}{c}\% \\
\mathrm{APD}\end{array}$ \\
\hline
\end{tabular}

\section{England}

B Heart

B QEH

Basldn

Bradfd

Brightn

Bristol

Camb

Carlis

Carsh

Chelms

Covnt

Derby

Donc

Dorset

Dudley

Exeter

Glouc

Hull

Ipswi

Kent

L Barts

L Guys

L Kings

L St.G

L West

Leeds

Leic

Liv Ain

Liv RI

M RI

Middlbr

Newc

Norwch

Nottm

Oxford

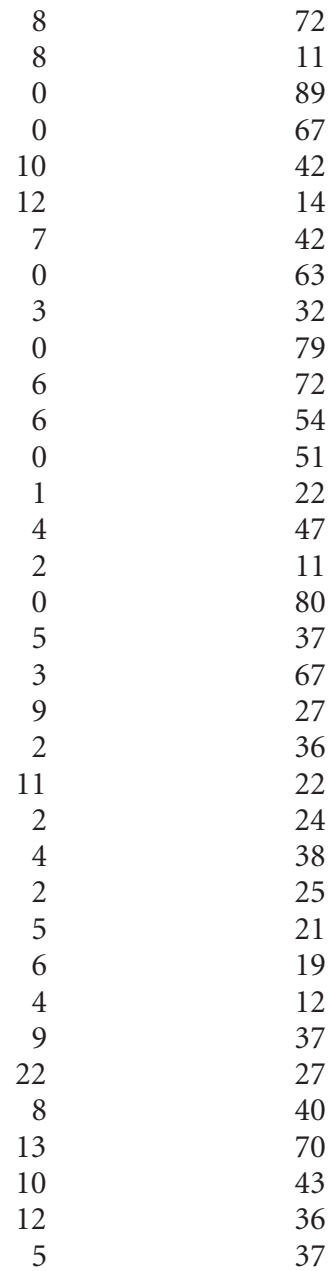

72

89

67

2

2

4

42

$36 \quad 31$

Oxford


Table F.2.8. Continued

\begin{tabular}{|c|c|c|c|c|c|c|}
\hline Centre & $\begin{array}{c}\% \\
\text { home HD }\end{array}$ & $\begin{array}{c}\% \\
\text { hospital HD }\end{array}$ & $\begin{array}{c}\% \\
\text { satellite HD }\end{array}$ & $\begin{array}{c}\% \\
\text { CAPD }\end{array}$ & $\begin{array}{l}\% \text { unknown } \\
\text { type of PD }\end{array}$ & $\begin{array}{c}\% \\
\text { APD }\end{array}$ \\
\hline Plymth & 10 & 57 & 0 & 20 & 2 & 10 \\
\hline Ports & 1 & 21 & 61 & 16 & 0 & 0 \\
\hline Prestn & 10 & 15 & 61 & 4 & 0 & 9 \\
\hline Redng & 0 & 30 & 38 & 31 & 0 & 1 \\
\hline Salford & 9 & 35 & 34 & 11 & 0 & 11 \\
\hline Sheff & 13 & 34 & 43 & 9 & 0 & 0 \\
\hline Shrew & 12 & 42 & 29 & 16 & 0 & 0 \\
\hline Stevng & 10 & 48 & 33 & 9 & 0 & 0 \\
\hline Sthend & 2 & 77 & 0 & 21 & 0 & 0 \\
\hline Stoke & 7 & 49 & 27 & 2 & 0 & 14 \\
\hline Sund & 2 & 68 & 18 & 7 & 0 & 5 \\
\hline Truro & 0 & 62 & 22 & 0 & 0 & 16 \\
\hline Wirral & 0 & 68 & 15 & 0 & 0 & 17 \\
\hline Wolve & 6 & 24 & 47 & 22 & 0 & 0 \\
\hline York & 12 & 48 & 21 & 19 & 0 & 0 \\
\hline \multicolumn{7}{|l|}{$\mathrm{N}$ Ireland } \\
\hline Antrim & 7 & 72 & 0 & 3 & 0 & 17 \\
\hline Belfast & 8 & 81 & 0 & 1 & 1 & 10 \\
\hline Newry & 0 & 86 & 0 & 0 & 0 & 14 \\
\hline Ulster & 19 & 71 & 0 & 0 & 0 & 10 \\
\hline West NI & 5 & 80 & 0 & 2 & 0 & 14 \\
\hline \multicolumn{7}{|l|}{ Scotland ${ }^{*}$} \\
\hline Abrdn & 4 & 80 & 0 & 6 & 0 & 9 \\
\hline Airdrie & 0 & 92 & 0 & 4 & 0 & 4 \\
\hline D \& Gall & 5 & 84 & 0 & 5 & 0 & 5 \\
\hline Dundee & 0 & 83 & 0 & 5 & 0 & 12 \\
\hline Dunfn & 0 & 83 & 0 & 0 & 0 & 17 \\
\hline Edinb & 3 & 84 & 0 & 2 & 0 & 10 \\
\hline Glasgw & 10 & 81 & 0 & 2 & 0 & 6 \\
\hline Inverns & 10 & 61 & 0 & 13 & 0 & 16 \\
\hline Klmarnk & 5 & 66 & 0 & 3 & 0 & 26 \\
\hline \multicolumn{7}{|l|}{ Wales } \\
\hline Bangor & 23 & 47 & 12 & 5 & 0 & 14 \\
\hline Cardff & 12 & 22 & 45 & 14 & 0 & 7 \\
\hline Clwyd & 3 & 83 & 0 & 10 & 0 & 3 \\
\hline Swanse & 14 & 47 & 18 & 15 & 0 & 5 \\
\hline Wrexm & 0 & 68 & 5 & 26 & 0 & 0 \\
\hline England & 7 & 35 & 41 & 8 & 0 & 9 \\
\hline N Ireland & 7 & 80 & 0 & 1 & 0 & 12 \\
\hline Scotland & 5 & 81 & 0 & 3 & 0 & 11 \\
\hline Wales & 12 & 40 & 27 & 14 & 0 & 6 \\
\hline UK & 7 & 41 & 36 & 8 & 0 & 9 \\
\hline
\end{tabular}

Excluded centres with $\geqslant 40 \%$ primary renal diagnosis aetiology uncertain (Colchester) as well as centres with $\geqslant 50 \%$ primary renal diagnosis not sent (London Royal Free)

Diabetic patients are patients with a primary renal disease code of diabetes

Non-diabetic patients are calculated as all patients excluding patients with diabetes and patients with a missing primary renal disease code

${ }^{*}$ All haemodialysis patients in centres in Scotland are shown as receiving treatment at home or in centre as no data is available regarding satellite dialysis 
Table F.2.9. Number of 2011 prevalent patients without diabetes aged under 65 by treatment modality

\begin{tabular}{lrrr}
\hline & HD & PD & Transplant \\
\hline England & 6,529 & 1,339 & 15,038 \\
N Ireland & 234 & 37 & 535 \\
Scotland & 732 & 118 & 1,669 \\
Wales & 338 & 86 & 966 \\
UK & $\mathbf{7 , 8 3 3}$ & $\mathbf{1 , 5 8 0}$ & $\mathbf{1 8 , 2 0 8}$ \\
\hline
\end{tabular}

Excluded centres with $\geqslant 40 \%$ primary renal diagnosis aetiology uncertain (Colchester) as well as centres with $\geqslant 50 \%$ primary renal diagnosis not sent (London Royal Free)

Diabetic patients are patients with a primary renal disease code of diabetes

Non-diabetic patients are calculated as all patients excluding patients with diabetes and patients with a missing primary renal disease code

Table F.2.10. Dialysis modalities for 2011 prevalent patients without diabetes aged over 65

\begin{tabular}{|c|c|c|c|c|c|c|}
\hline Centre & $\begin{array}{c}\% \\
\text { home HD }\end{array}$ & $\begin{array}{c}\% \\
\text { hospital HD }\end{array}$ & $\begin{array}{c}\% \\
\text { satellite HD }\end{array}$ & $\begin{array}{c}\% \\
\text { CAPD }\end{array}$ & $\begin{array}{l}\% \text { unknown } \\
\text { type of PD }\end{array}$ & $\begin{array}{c}\% \\
\mathrm{APD}\end{array}$ \\
\hline
\end{tabular}

\section{England}

B Heart

B QEH

Basldn

Bradfd

Brightn

Bristol

Camb

Carlis

Carsh

Chelms

Covnt

Derby

Donc

Dorset

Dudley

Exeter

Glouc

Hull

Ipswi

Kent

L Barts

L Guys

L Kings

L St.G

L West

Leeds

Leic

Liv Ain

Liv RI

M RI

$\begin{array}{rr}1 & 84 \\ 2 & 9 \\ 0 & 86 \\ 0 & 63 \\ 3 & 44 \\ 3 & 12 \\ 0 & 37 \\ 0 & 47 \\ 1 & 32 \\ 0 & 80 \\ 0 & 82 \\ 6 & 64 \\ 0 & 45 \\ 1 & 18 \\ 0 & 48 \\ 0 & 9 \\ 1 & 86 \\ 1 & 37 \\ 3 & 65 \\ 3 & 29 \\ 0 & 23 \\ 1 & 21 \\ 1 & 13 \\ 0 & 41 \\ 0 & 23 \\ 0 & 16 \\ 2 & 16 \\ 2 & 14 \\ 2 & 37 \\ 2 & 24\end{array}$

$\begin{array}{rr}10 & 6 \\ 77 & 6 \\ 1 & 8 \\ 28 & 1 \\ 35 & 11 \\ 76 & 7 \\ 55 & 0 \\ 27 & 18 \\ 57 & 3 \\ 0 & 13 \\ 0 & 18 \\ 0 & 27 \\ 47 & 0 \\ 62 & 7 \\ 29 & 15 \\ 75 & 11 \\ 0 & 5 \\ 47 & 6 \\ 13 & 14 \\ 57 & 12 \\ 60 & 8 \\ 72 & 4 \\ 72 & 9 \\ 43 & 7 \\ 74 & 1 \\ 72 & 3 \\ 66 & 6 \\ 75 & 2 \\ 49 & 8 \\ 58 & 2\end{array}$

0
6
5
8
7
2
0
9
7
6
0
3
8
1
9
5
9
10
4
0
8
3
6
7
1
8
10
6
4
3


Table F.2.10. Continued

\begin{tabular}{|c|c|c|c|c|c|c|}
\hline Centre & $\begin{array}{c}\% \\
\text { home HD }\end{array}$ & $\begin{array}{c}\% \\
\text { hospital HD }\end{array}$ & $\begin{array}{c}\% \\
\text { satellite HD }\end{array}$ & $\begin{array}{c}\% \\
\text { CAPD }\end{array}$ & $\begin{array}{l}\% \text { unknown } \\
\text { type of PD }\end{array}$ & $\begin{array}{c}\% \\
\text { APD }\end{array}$ \\
\hline Middlbr & 2 & 24 & 71 & 3 & 0 & 0 \\
\hline Newc & 1 & 85 & 0 & 3 & 0 & 11 \\
\hline Norwch & 2 & 48 & 38 & 8 & 0 & 3 \\
\hline Nottm & 2 & 34 & 46 & 10 & 0 & 7 \\
\hline Oxford & 2 & 38 & 42 & 8 & 0 & 10 \\
\hline Plymth & 0 & 77 & 0 & 20 & 0 & 3 \\
\hline Ports & 0 & 16 & 68 & 15 & 0 & 0 \\
\hline Prestn & 3 & 14 & 73 & 3 & 0 & 7 \\
\hline Redng & 1 & 46 & 36 & 17 & 0 & 0 \\
\hline Salford & 1 & 39 & 47 & 9 & 0 & 3 \\
\hline Sheff & 2 & 39 & 50 & 9 & 0 & 0 \\
\hline Shrew & 0 & 55 & 31 & 14 & 0 & 0 \\
\hline Stevng & 2 & 48 & 43 & 7 & 0 & 0 \\
\hline Sthend & 2 & 91 & 0 & 8 & 0 & 0 \\
\hline Stoke & 2 & 55 & 21 & 7 & 0 & 15 \\
\hline Sund & 0 & 63 & 30 & 3 & 0 & 4 \\
\hline Truro & 0 & 37 & 45 & 7 & 0 & 10 \\
\hline Wirral & 0 & 74 & 21 & 0 & 0 & 5 \\
\hline Wolve & 1 & 17 & 65 & 18 & 0 & 0 \\
\hline York & 0 & 56 & 30 & 14 & 0 & 0 \\
\hline \multicolumn{7}{|l|}{$\mathrm{N}$ Ireland } \\
\hline Antrim & 0 & 91 & 0 & 3 & 0 & 6 \\
\hline Belfast & 3 & 86 & 0 & 1 & 0 & 10 \\
\hline Newry & 4 & 85 & 0 & 0 & 0 & 11 \\
\hline Ulster & 0 & 100 & 0 & 0 & 0 & 0 \\
\hline West NI & 1 & 91 & 0 & 0 & 0 & 8 \\
\hline \multicolumn{7}{|l|}{ Scotland ${ }^{\star}$} \\
\hline Abrdn & 0 & 96 & 0 & 4 & 0 & 0 \\
\hline Airdrie & 0 & 96 & 0 & 0 & 0 & 4 \\
\hline D \& Gall & 0 & 73 & 0 & 9 & 0 & 18 \\
\hline Dundee & 0 & 90 & 0 & 0 & 0 & 10 \\
\hline Dunfn & 0 & 82 & 0 & 0 & 0 & 18 \\
\hline Edinb & 0 & 84 & 0 & 7 & 0 & 10 \\
\hline Glasgw & 1 & 93 & 0 & 2 & 0 & 3 \\
\hline Inverns & 2 & 80 & 0 & 6 & 0 & 12 \\
\hline Klmarnk & 4 & 78 & 0 & 2 & 0 & 16 \\
\hline \multicolumn{7}{|l|}{ Wales } \\
\hline Bangor & 4 & 56 & 17 & 8 & 0 & 15 \\
\hline Cardff & 1 & 14 & 70 & 10 & 0 & 5 \\
\hline Clwyd & 4 & 88 & 0 & 8 & 0 & 0 \\
\hline Swanse & 3 & 50 & 36 & 8 & 0 & 1 \\
\hline Wrexm & 0 & 82 & 4 & 14 & 0 & 0 \\
\hline England & 1 & 36 & 50 & 8 & 0 & 5 \\
\hline N Ireland & 2 & 90 & 0 & 1 & 0 & 7 \\
\hline Scotland & 1 & 88 & 0 & 3 & 0 & 8 \\
\hline Wales & 2 & 39 & 45 & 10 & 0 & 4 \\
\hline UK & 1 & 42 & 44 & 7 & 0 & 5 \\
\hline
\end{tabular}

Excluded centres with $\geqslant 40 \%$ primary renal diagnosis aetiology uncertain (Colchester) as well as centres with $\geqslant 50 \%$ primary renal diagnosis not sent (London Royal Free)

Diabetic patients are patients with a primary renal disease code of diabetes

Non-diabetic patients are calculated as all patients excluding patients with diabetes and patients with a missing primary renal disease code

* All haemodialysis patients in centres in Scotland are shown as receiving treatment at home or in centre as no data is available regarding satellite dialysis 
Table F.2.11. Number of 2011 prevalent patients without diabetes aged over 65 by treatment modality

\begin{tabular}{lrrc}
\hline & HD & PD & Transplant \\
\hline England & 7,472 & 1,109 & 3,497 \\
N Ireland & 324 & 28 & 113 \\
Scotland & 752 & 94 & 336 \\
Wales & 512 & 80 & 243 \\
UK & $\mathbf{9 , 0 6 0}$ & $\mathbf{1 , 3 1 1}$ & $\mathbf{4 , 1 8 9}$ \\
\hline
\end{tabular}

Excluded centres with $\geqslant 40 \%$ primary renal diagnosis aetiology uncertain (Colchester) as well as centres with $\geqslant 50 \%$ primary renal diagnosis not sent (London Royal Free)

Diabetic patients are patients with a primary renal disease code of diabetes

Non-diabetic patients are calculated as all patients excluding patients with diabetes and patients with a missing primary renal disease code

Table F.2.12. Dialysis modalities for 2011 prevalent patients with diabetes

\begin{tabular}{|c|c|c|c|c|c|c|}
\hline Centre & $\begin{array}{c}\% \\
\text { home HD }\end{array}$ & $\begin{array}{c}\% \\
\text { hospital HD }\end{array}$ & $\begin{array}{c}\% \\
\text { satellite HD }\end{array}$ & $\begin{array}{c}\% \\
\text { CAPD }\end{array}$ & $\begin{array}{l}\% \text { unknown } \\
\text { type of PD }\end{array}$ & $\begin{array}{c}\% \\
\mathrm{APD}\end{array}$ \\
\hline
\end{tabular}

\section{England}

B Heart

B QEH

Basldn

Bradfd

Brightn

Bristol

Camb

Carlis

Carsh

Chelms

Covnt

Derby

Donc

Dorset

Dudley

Exeter

Glouc

Hull

Ipswi

Kent

L Barts

L Guys

L Kings

L St.G

L West

Leeds

Leic

Liv Ain

Liv RI

M RI

Middlbr

Newc

Norwch

$\begin{array}{rr}5 & 82 \\ 2 & 12 \\ 0 & 77 \\ 0 & 90 \\ 9 & 27 \\ 3 & 20 \\ 4 & 40 \\ 0 & 59 \\ 0 & 26 \\ 0 & 93 \\ 4 & 77 \\ 5 & 57 \\ 3 & 49 \\ 0 & 26 \\ 2 & 50 \\ 0 & 9 \\ 0 & 76 \\ 0 & 32 \\ 4 & 65 \\ 0 & 45 \\ 1 & 32 \\ 1 & 33 \\ 0 & 20 \\ 1 & 44 \\ 0 & 25 \\ 0 & 22 \\ 2 & 24 \\ 0 & 28 \\ 2 & 38 \\ 2 & 41 \\ 0 & 38 \\ 0 & 89 \\ 0 & 52 \\ & \end{array}$

$\begin{array}{rr}6 & 4 \\ 76 & 3 \\ 0 & 9 \\ 5 & 0 \\ 55 & 3 \\ 69 & 2 \\ 44 & 0 \\ 0 & 12 \\ 62 & 5 \\ 0 & 0 \\ 0 & 19 \\ 0 & 28 \\ 26 & 3 \\ 58 & 5 \\ 14 & 24 \\ 77 & 4 \\ 0 & 6 \\ 36 & 12 \\ 15 & 8 \\ 37 & 18 \\ 52 & 8 \\ 62 & 2 \\ 63 & 5 \\ 43 & 2 \\ 73 & 1 \\ 67 & 3 \\ 63 & 4 \\ 70 & 0 \\ 38 & 6 \\ 43 & 4 \\ 58 & 4 \\ 0 & \\ 36 & 2 \\ & \\ 53 \\ 53\end{array}$

$\begin{array}{rr}0 & 3 \\ 0 & 7 \\ 0 & 14 \\ 0 & 5 \\ 0 & 6 \\ 0 & 5 \\ 8 & 4 \\ 0 & 29 \\ 0 & 7 \\ 0 & 7 \\ 0 & 0 \\ 0 & 11 \\ 0 & 20 \\ 0 & 12 \\ 0 & 10 \\ 0 & 10 \\ 0 & 18 \\ 0 & 20 \\ 0 & 8 \\ 0 & 0 \\ 0 & 8 \\ 0 & 2 \\ 0 & 12 \\ 0 & 11 \\ 0 & 1 \\ 0 & 9 \\ 0 & 8 \\ 0 & 3 \\ 0 & 17 \\ 0 & 0 \\ 0 & \\ 0 & 9 \\ 0 & 0 \\ & 3 \\ 0 & 0\end{array}$


Table F.2.12. Dialysis modalities for 2011 prevalent patients with diabetes

\begin{tabular}{|c|c|c|c|c|c|c|}
\hline Centre & $\begin{array}{c}\% \\
\text { home HD }\end{array}$ & $\begin{array}{c}\% \\
\text { hospital HD }\end{array}$ & $\begin{array}{c}\% \\
\text { satellite HD }\end{array}$ & $\begin{array}{c}\% \\
\text { CAPD }\end{array}$ & $\begin{array}{l}\% \text { unknown } \\
\text { type of PD }\end{array}$ & $\begin{array}{c}\% \\
\text { APD }\end{array}$ \\
\hline Nottm & 5 & 51 & 26 & 8 & 0 & 10 \\
\hline Oxford & 4 & 36 & 43 & 4 & 0 & 13 \\
\hline Plymth & 0 & 73 & 0 & 24 & 0 & 2 \\
\hline Ports & 0 & 30 & 58 & 12 & 0 & 0 \\
\hline Prestn & 1 & 27 & 65 & 3 & 0 & 5 \\
\hline Redng & 0 & 33 & 41 & 25 & 0 & 1 \\
\hline Salford & 2 & 24 & 51 & 15 & 0 & 8 \\
\hline Sheff & 3 & 36 & 50 & 12 & 0 & 0 \\
\hline Shrew & 2 & 48 & 31 & 19 & 0 & 0 \\
\hline Stevng & 2 & 50 & 47 & 2 & 0 & 0 \\
\hline Sthend & 3 & 86 & 0 & 10 & 0 & 0 \\
\hline Stoke & 2 & 60 & 12 & 2 & 0 & 22 \\
\hline Sund & 0 & 74 & 20 & 3 & 0 & 3 \\
\hline Truro & 3 & 61 & 29 & 5 & 0 & 3 \\
\hline Wirral & 0 & 63 & 22 & 6 & 0 & 9 \\
\hline Wolve & 1 & 33 & 50 & 16 & 0 & 0 \\
\hline York & 0 & 50 & 39 & 11 & 0 & 0 \\
\hline \multicolumn{7}{|l|}{$\mathrm{N}$ Ireland } \\
\hline Antrim & 4 & 92 & 0 & 2 & 0 & 2 \\
\hline Belfast & 6 & 81 & 0 & 2 & 0 & 10 \\
\hline Newry & 3 & 94 & 0 & 0 & 0 & 3 \\
\hline Ulster & 0 & 95 & 0 & 0 & 0 & 5 \\
\hline West NI & 0 & 89 & 0 & 0 & 0 & 11 \\
\hline \multicolumn{7}{|l|}{ Scotland ${ }^{*}$} \\
\hline Abrdn & 0 & 92 & 0 & 2 & 0 & 6 \\
\hline Airdrie & 0 & 97 & 0 & 0 & 0 & 3 \\
\hline D \& Gall & 0 & 73 & 0 & 0 & 0 & 27 \\
\hline Dundee & 0 & 96 & 0 & 2 & 0 & 2 \\
\hline Dunfn & 0 & 90 & 0 & 0 & 0 & 10 \\
\hline Edinb & 2 & 88 & 0 & 5 & 0 & 6 \\
\hline Glasgw & 2 & 91 & 0 & 2 & 0 & 6 \\
\hline Inverns & 5 & 95 & 0 & 0 & 0 & 0 \\
\hline Klmarnk & 0 & 77 & 0 & 0 & 0 & 23 \\
\hline \multicolumn{7}{|l|}{ Wales } \\
\hline Bangor & 6 & 56 & 28 & 0 & 0 & 11 \\
\hline Cardff & 4 & 15 & 66 & 14 & 0 & 1 \\
\hline Clwyd & 13 & 75 & 0 & 13 & 0 & 0 \\
\hline Swanse & 3 & 53 & 28 & 10 & 0 & 5 \\
\hline Wrexm & 5 & 74 & 5 & 16 & 0 & 0 \\
\hline England & 1 & 38 & 48 & 7 & 0 & 6 \\
\hline $\mathrm{N}$ Ireland & 3 & 89 & 0 & 1 & 0 & 6 \\
\hline Scotland & 1 & 90 & 0 & 2 & 0 & 7 \\
\hline Wales & 4 & 37 & 44 & 12 & 0 & 3 \\
\hline UK & 2 & 44 & 42 & 6 & 0 & 6 \\
\hline
\end{tabular}

Excluded centres with $\geqslant 40 \%$ primary renal diagnosis aetiology uncertain (Colchester) as well as centres with $\geqslant 50 \%$ primary renal diagnosis not sent (London Royal Free)

Diabetic patients are patients with a primary renal disease code of diabetes

Non-diabetic patients are calculated as all patients excluding patients with diabetes and patients with a missing primary renal disease code ${ }^{*}$ All haemodialysis patients in centres in Scotland are shown as receiving treatment at home or in centre as no data is available regarding satellite dialysis 
Table F.2.13. Number of 2011 prevalent patients with diabetes by treatment modality

\begin{tabular}{lrrr}
\hline & HD & PD & Transplant \\
\hline England & 3,960 & 595 & 1,959 \\
N Ireland & 167 & 13 & 55 \\
Scotland & 388 & 37 & 189 \\
Wales & 240 & 42 & 153 \\
UK & $\mathbf{4 , 7 5 5}$ & $\mathbf{6 8 7}$ & $\mathbf{2 , 3 5 6}$ \\
\hline
\end{tabular}

Excluded centres with $\geqslant 40 \%$ primary renal diagnosis aetiology uncertain (Colchester) as well as centres with $\geqslant 50 \%$ primary renal diagnosis not sent (London Royal Free)

Diabetic patients are patients with a primary renal disease code of diabetes

Non-diabetic patients are calculated as all patients excluding patients with diabetes and patients with a missing primary renal disease code

Table F.2.14. With diabetes 2011 prevalent patients

\begin{tabular}{|c|c|c|c|c|c|}
\hline Centre & $\mathrm{M}: \mathrm{F}$ ratio & $\begin{array}{c}\text { Median age on } \\
31 / 12 / 2011\end{array}$ & $\begin{array}{l}\text { Median age at } \\
\text { start of treatment }\end{array}$ & $\begin{array}{l}\text { Median time on } \\
\text { RRT in days }\end{array}$ & $\begin{array}{l}\text { Median time on } \\
\text { RRT in years }\end{array}$ \\
\hline
\end{tabular}

\section{England}

\section{B Heart}

$\mathrm{B}$ QEH

Basldn

Bradfd

Brightn

Bristol

Camb

Carlis

Carsh

Chelms

Covnt

Derby

Donc

Dorset

Dudley

Exeter

Glouc

Hull

Ipswi

Kent

L Barts

L Guys

L Kings

L St.G

L West

Leeds

Leic

Liv Ain

Liv RI

M RI

Middlbr

Newc

$\begin{array}{ll}1.8 & 67\end{array}$

1.4

2.9

1.5

1.9

1.3

1.9

3.5

2.1

2.4

2.2

$$
1.8
$$

3.4

1.4

\section{3}

1.5

1.5

1.7

1.6

1.2

1.3

1.3

1.9

2.0

1.5

1.4

1.4

1.4

67

63

61

62

62

48

59

64

61

63

66

62

62

62
62

63

60

59

59

63

57

64

67

63

62

63

61

55

57

54

1.1
1.3
62

58

57

54

54

55

40

58

60

58

59

61

58

56

59

58

59

54

53

57

59

52

59

62

58

56

58

57

47

53

47

50

$$
\begin{array}{r}
1,034 \\
1,333 \\
1,129 \\
1,218 \\
1,888 \\
1,431 \\
1,997 \\
694 \\
1,379 \\
798 \\
1,296 \\
1,251 \\
840 \\
1,207 \\
1,376 \\
956 \\
1,489 \\
1,493 \\
985 \\
879 \\
1,075 \\
1,716 \\
1,060 \\
1,319 \\
1,275 \\
1,419 \\
1,113 \\
921 \\
2,056 \\
1,447 \\
1,301 \\
1,552 \\
\\
\hline
\end{array}
$$


Table F.2.14. With diabetes 2011 prevalent patients

\begin{tabular}{|c|c|c|c|c|c|}
\hline Centre & M:F ratio & $\begin{array}{c}\text { Median age on } \\
31 / 12 / 2011\end{array}$ & $\begin{array}{l}\text { Median age at } \\
\text { start of treatment }\end{array}$ & $\begin{array}{l}\text { Median time on } \\
\text { RRT in days }\end{array}$ & $\begin{array}{l}\text { Median time on } \\
\text { RRT in years }\end{array}$ \\
\hline Norwch & 1.4 & 61 & 58 & 1,257 & 3.4 \\
\hline Nottm & 1.5 & 60 & 54 & 1,517 & 4.2 \\
\hline Oxford & 1.9 & 55 & 49 & 1,240 & 3.4 \\
\hline Plymth & 1.6 & 58 & 54 & 1,319 & 3.6 \\
\hline Ports & 2.0 & 59 & 54 & 1,125 & 3.1 \\
\hline Prestn & 1.8 & 63 & 59 & 1,013 & 2.8 \\
\hline Redng & 1.5 & 63 & 60 & 1,131 & 3.1 \\
\hline Salford & 1.2 & 65 & 59 & 1,039 & 2.8 \\
\hline Sheff & 2.5 & 60 & 56 & 1,206 & 3.3 \\
\hline Shrew & 1.3 & 63 & 62 & 794 & 2.2 \\
\hline Stevng & 2.1 & 63 & 60 & 1,121 & 3.1 \\
\hline Sthend & 1.1 & 63 & 59 & 1,392 & 3.8 \\
\hline Stoke & 1.2 & 65 & 61 & 1,081 & 3.0 \\
\hline Sund & 2.1 & 58 & 54 & 938 & 2.6 \\
\hline Truro & 2.0 & 57 & 52 & 1,416 & 3.9 \\
\hline Wirral & 1.2 & 58 & 54 & 1,396 & 3.8 \\
\hline Wolve & 2.0 & 62 & 58 & 1,289 & 3.5 \\
\hline York & 1.2 & 57 & 53 & 1,058 & 2.9 \\
\hline \multicolumn{6}{|l|}{ N Ireland } \\
\hline Antrim & 1.0 & 65 & 63 & 1,437 & 3.9 \\
\hline Belfast & 1.7 & 56 & 50 & 1,387 & 3.8 \\
\hline Newry & 2.3 & 67 & 60 & 670 & 1.8 \\
\hline Ulster & 1.7 & 69 & 65 & 695 & 1.9 \\
\hline West NI & 0.9 & 64 & 59 & 1,493 & 4.1 \\
\hline \multicolumn{6}{|l|}{ Scotland } \\
\hline Abrdn & 1.8 & 58 & 53 & 1,360 & 3.7 \\
\hline Airdrie & 1.7 & 55 & 52 & 788 & 2.2 \\
\hline D \& Gall & 4.0 & 55 & 45 & 928 & 2.5 \\
\hline Dundee & 1.3 & 60 & 56 & 933 & 2.6 \\
\hline Dunfn & 1.2 & 58 & 57 & 1,009 & 2.8 \\
\hline Edinb & 1.3 & 52 & 47 & 1,478 & 4.0 \\
\hline Glasgw & 1.4 & 60 & 55 & 1,287 & 3.5 \\
\hline Inverns & 2.5 & 51 & 43 & 2,208 & 6.0 \\
\hline Klmarnk & 1.7 & 58 & 54 & 1,192 & 3.3 \\
\hline \multicolumn{6}{|l|}{ Wales } \\
\hline Bangor & 1.3 & 65 & 62 & 988 & 2.7 \\
\hline Cardff & 1.5 & 60 & 54 & 1,147 & 3.1 \\
\hline Clwyd & 0.9 & 57 & 50 & 1,706 & 4.7 \\
\hline Swanse & 2.0 & 64 & 61 & 841 & 2.3 \\
\hline Wrexm & 2.8 & 54 & 42 & 2,696 & 7.4 \\
\hline England & 1.6 & 61 & 56 & 1,261 & 3.5 \\
\hline N Ireland & 1.4 & 63 & 59 & 1,140 & 3.1 \\
\hline Scotland & 1.5 & 58 & 53 & 1,225 & 3.4 \\
\hline Wales & 1.7 & 61 & 55 & 1,143 & 3.1 \\
\hline UK & 1.6 & 61 & 56 & 1,249 & 3.4 \\
\hline
\end{tabular}

Excluded centres with $\geqslant 40 \%$ primary renal diagnosis aetiology uncertain (Colchester) as well as centres with $\geqslant 50 \%$ primary renal diagnosis not sent (London Royal Free)

Diabetic patients are patients with a primary renal disease code of diabetes

Non-diabetic patients are calculated as all patients excluding patients with diabetes and patients with a missing primary renal disease code 
Table F.2.15. Transplant gender ratios in 2011 prevalent patients

\begin{tabular}{lccrrr}
\hline & \% males & \% females & N males & N females & M:F ratio \\
\hline England & 60.7 & 39.3 & 13,354 & 8,635 & 1.5 \\
N Ireland & 63.6 & 36.4 & 450 & 857 & 1.8 \\
Scotland & 59.9 & 40.1 & 1,315 & 513 & 1.5 \\
Wales & 62.7 & 37.3 & 863 & $\mathbf{1 5 , 9 8 2}$ & $\mathbf{1 0 , 2 8 7}$ \\
UK & $\mathbf{6 0 . 8}$ & $\mathbf{3 9 . 2}$ & & & $\mathbf{1 . 6}$ \\
\hline
\end{tabular}

